\title{
Utilización de fuels pesados en la fabricación de cementos
}

ANTONIO RUBIO REQUENA

A P L E S A

\section{INTRODUCCION}

A partir del comienzo de la década de los años sesenta y hasta finales de 1973, momento en el que se desencadenó la crisis energética, el desarrollo industrial de España fue sin duda muy notable. Factores decisivos de ese desarrollo se debieron a los progresos alcanzados en el automatismo y mecanización de los diferentes procesos industriales en general y, en particular, de los grandes hornos y calderas. También constituyó un factor básico del desarrollo la aparición y expansión en el mercado nacional de un combustible económico, fácilmente manejable y de elevado poder calorífico: el fuel-oil.

Hace años que se conocen, aunque con frecuencia se han interpretado equivocadamente, las íntimas correlaciones existentes entre la "renta per capita" y "el consumo de energía primaria" en los diversos países.

Uno de los primeros gráficos en el que se ponían de relieve estas correlaciones lo debemos al Profesor E. S. Mason, de la Universidad de Harvard, y se basa en datos del año 1952 (fig. 1). La recta de ajuste, trazada por Y. Mainguy, por simple estimación gráfica pone de manifiesto la proporcionalidad existente entre las citadas magnitudes: consumo de energía primaria por persona en unidades T.E.C. y "renta per capita" en los diferentes países.

En el año 1973, Hottel publicó un nuevo gráfico (fig. 2) con valores más recientes, pues se referían a datos del año 1969 e incluía la situación en 52 países. La curva de ajuste deducida por Hottel tiene por ecuación:

$$
Y=0,54 x^{0,80}
$$

representando en abscisas los consumos de energía primaria per capita en miles de $\mathrm{kWh}$ y, en ordenadas, las rentas nacionales per capita en miles de dólares U.S.A.

En lo que se refiere al año 1969 y en aquellas circunstancias, en un país cuya evolución se aproximase a la curva de ajuste de la figura 2 para duplicar la renta per capita era necesario multiplicar por 2,38 el consumo de energía primaria por habitante. Esta conclusión, que en principio puede parecer extraña, se justifica por el hecho de que el aumento en los bienes producidos, factor básico para la determinación de la renta per capita, no es el único que incide en el incremento del consumo energético. En este último participan igualmente las exigencias que lleva aparejadas la consecución de un mayor confort de vida: electrodomésticos, aire acondicionado, sistemas individuales de transporte, etc. 
En España, para duplicar la renta per capita, fenómeno que se produjo en el período de 13 años transcurridos desde 1960 a 1972, fue necesario multiplicar por 2,40 el consumo de energía primaria por habitante (fig. 3). Pero este índice ha presentado tendencias muy diferentes a lo largo de la etapa considerada, como se pone de manifiesto mediante el exa-

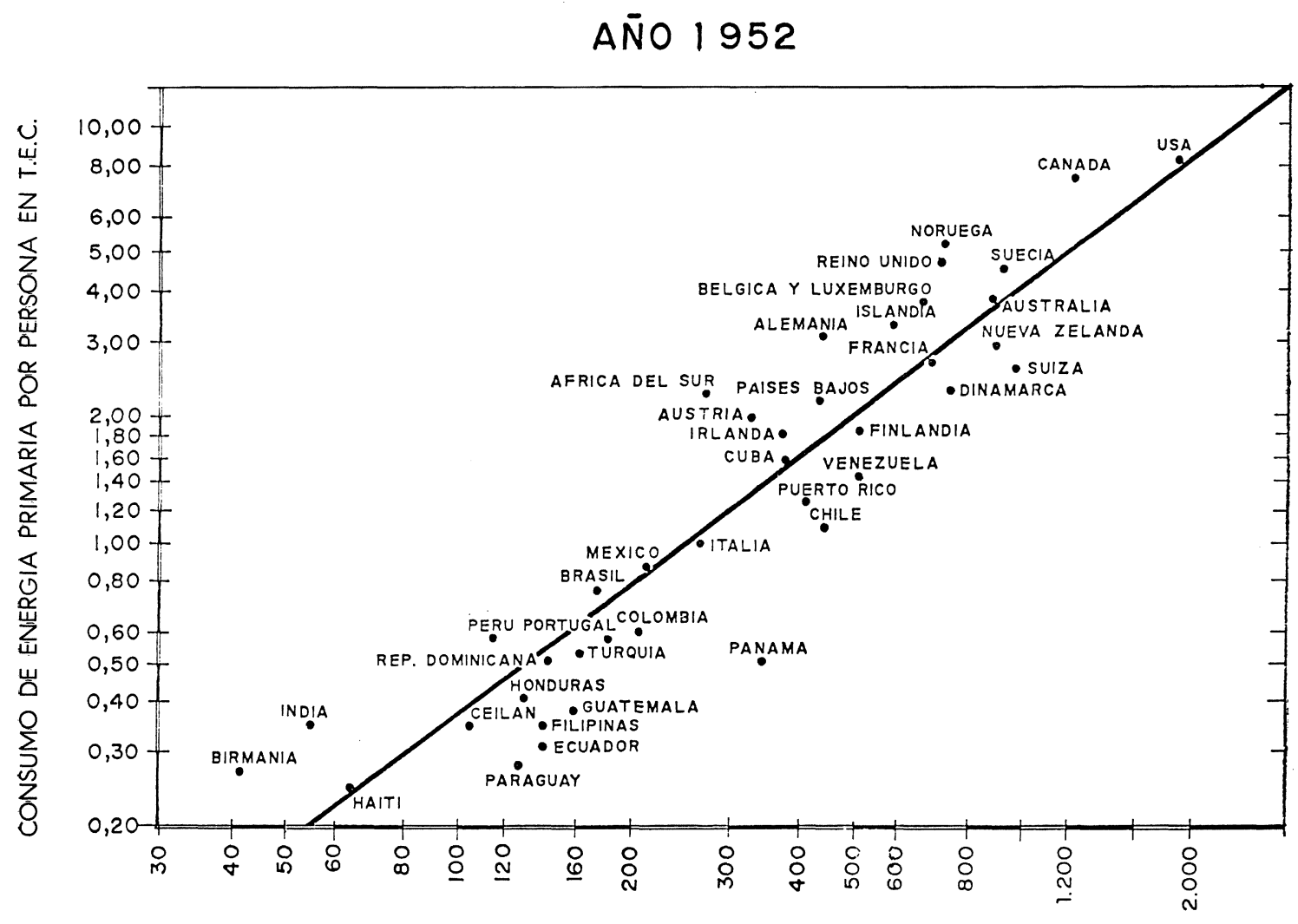

RENTA PER CAPITA EN DOLARES USA

Fig. 1

men de las rentas parciales de ajuste de la evolución experimentada en los subperíodos que comprenden los años de 1960 a 1966, por una parte, y de 1967 a 1972, por otra. Las circunstancias existentes en el desarrollo español del primer subperíodo (1960-1966), de haber persistido hasta 1972 nos habrían permitido duplicar nuestra renta per capita multiplicando tan sólo por 1,94 el consumo de energía primaria por habitante y año. En cuanto al segundo de los subperíodos mencionados las circunstancias fueron tales que para lograr duplicar la citada renta se hubiese hecho necesario multiplicar por 2,78 el consumo de energía primaria por persona y año.

Esta sustancial diferencia en la evolución energética española entre los dos períodos considerados pudo obedecer y de hecho se debió a una multiplicidad de factores. Mediada la década de los años sesenta se produjo en nuestro país la "explosión" del automovilismo y comenzaron a ser mejor atendidos los deseos de un mayorl confort en el sector doméstico. Se generalizó la utilización del butano en cocinas y calefacciones, que vino a desplazar casi totalmente a las leñas y carbones vegetales en las zonas rurales y urbanas y se inició el desarrollo de las instalaciones de calefacción centralizadas, las de agua caliente sanitaria y las de aire acondicionado. 
Pero aunque no pueda despreciarse la aportación de las causas mencionadas al desorbitante incremento en el consumo de energía primaria hay que buscar, para explicarnos satisfactoriamente este fenómeno, la causa determinante que vamos a tratar de analizar seguidamente.

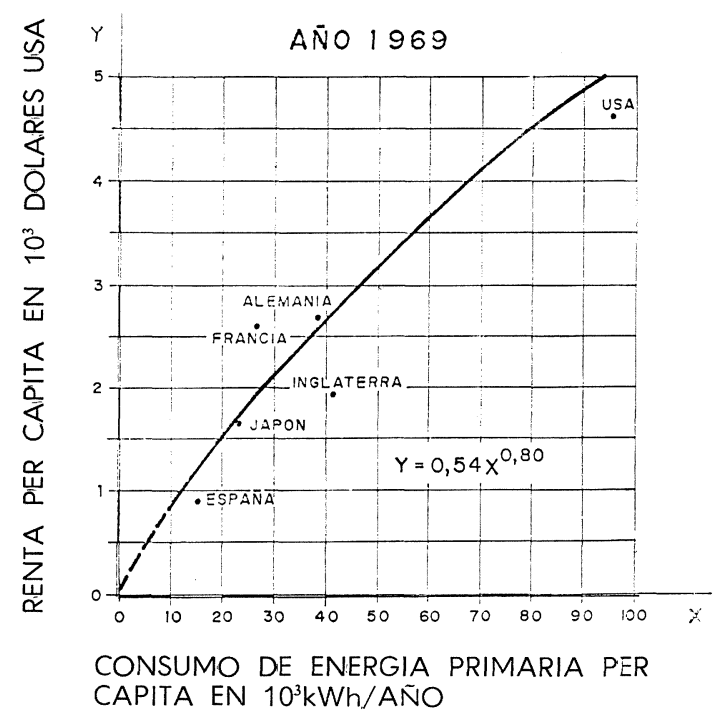

Fig. 2

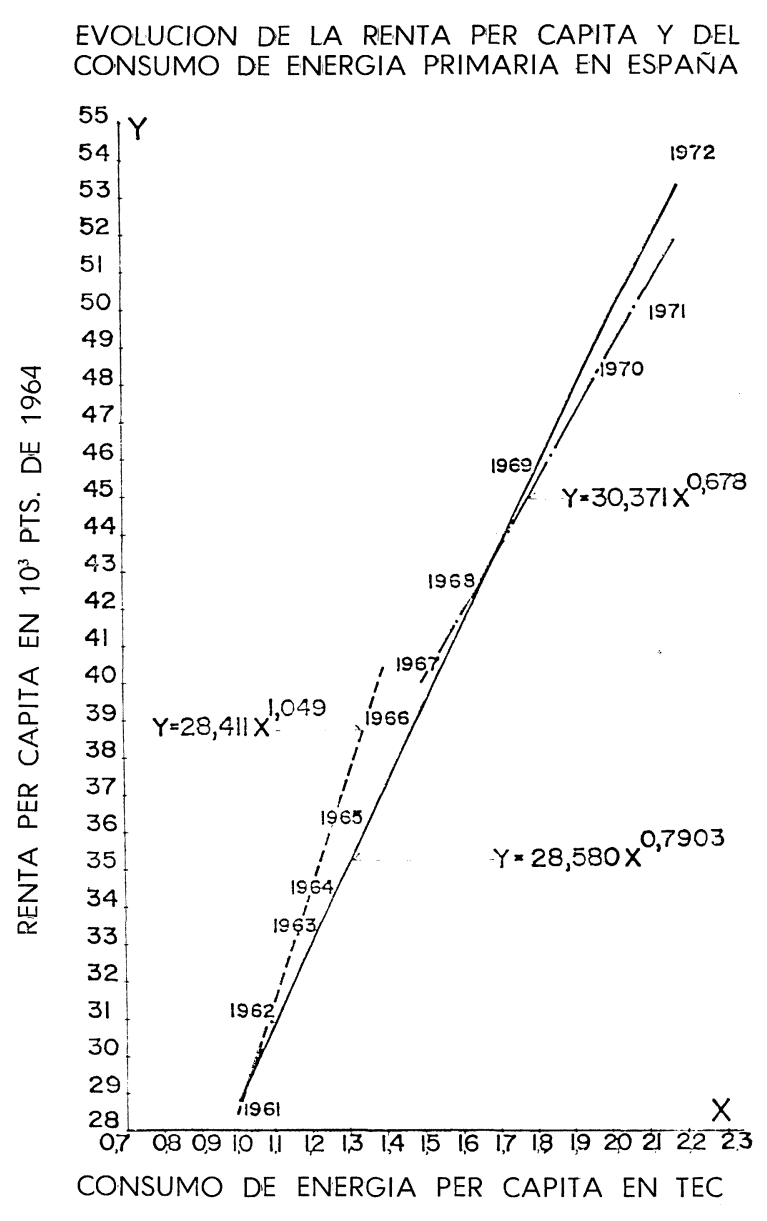

Fig. 3

Nuestra Addministración, deseosa de elevar el nivel de vida de los españoles, y tal vez por considerar como un axioma la correlación entre consumo de energía y renta nacional que venimos examinando, se inclinó, especialmente a partir de la mitad de la década de los sesenta, por seguir una política de energía barata, cada vez más económica y abundante, para lo que se hubo de recurrir a importaciones gradualmente más y más elevadas de crudos de petróleo extranjero (fig. 4). De este modo, el precio del fuel-oil a pesetas constantes, en el año 1973, tanto para usos generales como para el específico de la fabricación de cementos, apenas suponía un $40 \%$ del coste de dicho combustible quince años atrás.

En sus comienzos, esta línea de actuación de la Addminstración habría de calificarse como muy acertada. Eran tiempos de mano de obra abundante y barata, materias primas casi ilimitadas para el fenómeno de actividades fabriles como la fabricación de cementos, buenas perspectivas de exportación y un mercado potencial interno muy importante y en continuo progreso a medida que aumentaba nuestro poder adquisitivo. 
Lo equivocado - a mi juicio- de la política de energía barata es que se mantuvo y se continúa manteniendo a ultranza, por lo que en los últimos años está empezando a resultar evidentemente perjudicial para ia economía nacional. Conviene hacer algunos comentarios sobre los efectos de la política energética seguida en los últimos años.

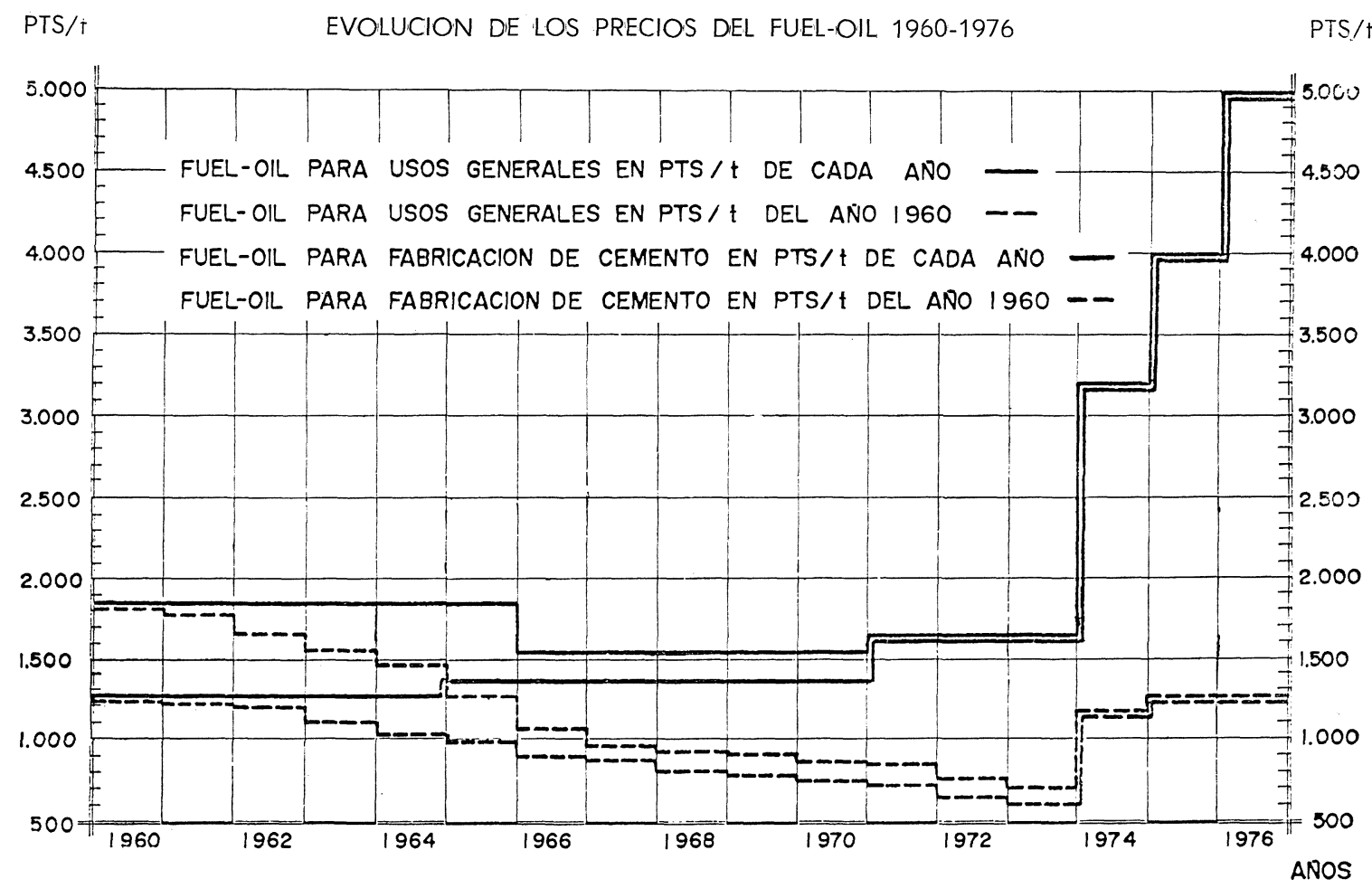

Fig. 4

En primer lugar hemos presenciado el desarrollo de una industria del refino simple, que procesa un gran volumen de crudos de petróleo, evidentemente excesivo para las posibilidades del país, y que obtiene un pequeño porcentaje de productos de calidad diferenciada arrojando al desecho, esto es al fuel-oil, cantidades muy superiores al $50 \%$ de su fabricación.

Por otra parte, la disponibilidad de crudos de petróleo baratos y abundantes restó interés en España por la explotación de nuestros propios recursos petrolíferos y por la investigación o desarrollo de nuevas fuentes energéticas y puso en graves apuros, ante la competencia del fuel-oil, a extensos sectores de la minería del carbón.

Otro problema que empezó a revestir caracteres graves, finalizando ya la década de los sesenta, especialmente en Madrid y en algunas áreas de gran concentración industrial, fue el de la progresiva contaminación del aire. Cierto que en este problema tuvieron su aportación defectos de consideración en la política de planificación y desarrollo de ciudades y zonas industriales, pero en buena parte sus efectos habían de achacarse a la política fiscal vigente durante muchos años que impedía la utilización en España de combustibles más limpios y de mejor calidad, de los que éramos excedentarios, y que se exportaban a bajo precio. 
Pero tal vez lo más lamentable de la política energética de los últimos lustros consis tió en sus efectos alentadores para el defectuoso aprovechamiento de la energía contenida en los combustibles, en su despilfarro en definitiva. Cierto que a cada situación económica corresponde un punto de equilibrio, entre el cosie del combustible y la amortización de las inversiones requeridas para llevar a cabo el aprovechamiento de la energía hasta un determinado grado. La política de combustible barato a ultranza ha conseguido transferir, en cierto modo, del sector privado al público, la realización de estas inversiones mediante la bonificación del precio del combustible. Esto ha constituido una ayuda estatal, aparentemente invisible, y a fondo perdido a los consumidores. Aunque naturalmente que la ayuda no ha ido a fondo perdido, sino a las arcas de los países exportadores de petróleo. Se privó, en consecuencia, a la economía nacional de un importante volumen de divisas cuyo equivalente en pesetas podría haber ido destinado a la industria nacional dedicada a la instalación de sistemas para un mejor aprovechamiento de la energía. Por otra parte, el mantenimiento de combustibles a precios bonificados obligó, como compensación en el interior de nuestras fronteras, a encarecer excesivamente el precio de los restantes derivados del petróleo, con perjuicios evidentes para sus usuarios y también para otros importantes sectores industriales como el del automóvil.

Del análisis de los principales sectores consumidores de combustibles se llega a la conclusión de que es posible economizar, en nuestro país, sobre un $30 \%$ del combustible utilizado y ello sin otros recursos que el de aplicar la tecnología ya existente y experimentada en los sistemas de recuperación del calor perdido, en los procesos, en los equipos y en las características y precios de los distintos combustibles que se puedan ofrecer como alternativa.

Un buen aprovechamiento de la energía requiere, con frecuencia, una actuación sobre los elementos de generación, transmisión y recuperación del calor en el dominio de la actividad fabril considerada. Sin entrar ahora en el análisis de las posibilidades directas de ahorro energético que se ofrecen a la industria del cemento, tema que es objeto de otra comunicación, trataremos de justificar la conveniencia de utilizar en este sector fuels de tipo pesado. Cierto que con esta sola medida no cabe esperar economías de combustible, pues habrían de seguir consumiéndose las mismas toneladas, pero sí otras ventajas entre las que habría de destacarse un posible menor precio.

\section{EL FUEL PESADO N. ${ }^{\circ} 2$}

Contrariamente a lo que sucede en los paísss que han alcanzado un cierto grado de desarrollo, en España el mercado del fuel-oil se caracteriza por la presencia en el mismo de un solo producto de carácter indiferenciado que puede presentar oscilaciones muy fuertes en su calidad y propiedades.

Pese a que por Decreto del Ministerio de Industria de 23 de agosto de 1975 se tipificaban en España las características y condiciones de empleo de los combustibles, estableciendo dos grados de fuel-oil pesado, los designados con los numeros 1 y 2 , lo cierto es que hasta el presente estos combustibles no se han puesto en el mercado. Se está actuando por consecuencia como si las necesidades y posibilidades de todos los consumidores de fuels, tanto en lo que se refiere a calidades como a precios y condiciones de empleo, fueran similares, lo que está muy lejos de la verdad.

Los elementos consumidores de fuel-oil para la generación de calor pueden calificarse de manera muy general según se indica en la tabla I. 


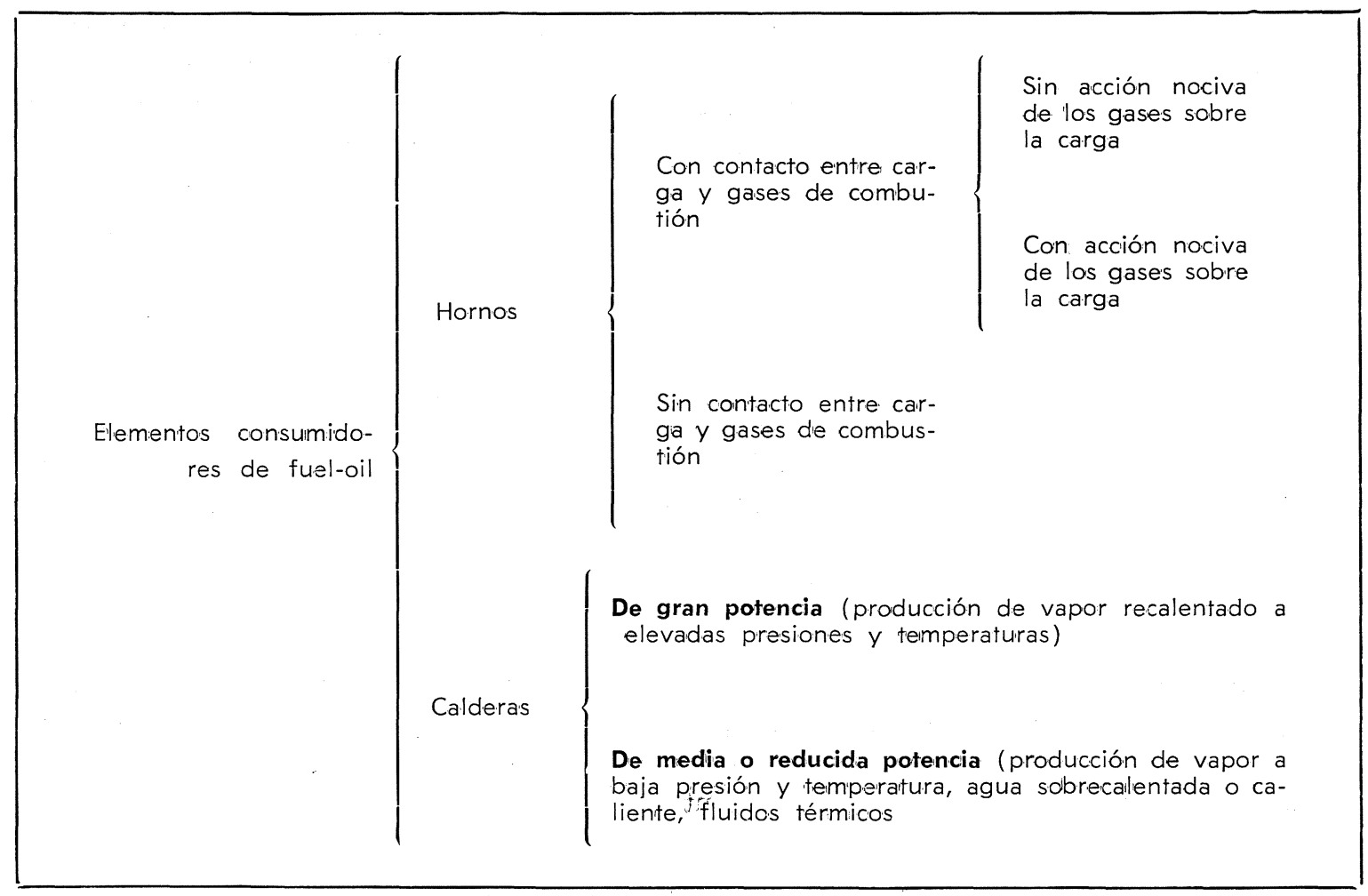

Son tres, en esencia, las propiedades físicas y químicas que caracterizan a un fuel: la viscosidad, el contenido en impurezas (azufre principalmente y también en menor grado $\mathrm{V}, \mathrm{Na}$ y $\mathrm{K}$ ) y el porcentaje de asfaltenos.

La viscosidad del combustible, aunque sea elevada, no presenta ningún problema serio para la utilización del mismo en grandes hornos y calderas. Por el contrario, en calderas de pequeña o mediana potencia, equipadas con quemadores de pulverización mecánica, una viscosidad excesiva o irregular en el fuel se traduce en pulverizaciones groseras, aparición de humos o inquemados, suciedad en las superficies de transferencia del calor y necesidad de trabajar con elevados excesos de aire que conducen a reducidos rendimientos térmicos.

En los hornos en los que la acción de los gases de la combustión no sea nociva sobre la carga y también en aquéllos en que no hay contacto entre gases y carga no importa el a.lcance del contenido de impurezas del combustible, salvo las corrosiones en canales de humos a baja temperatura ocasionadas por los ácidos derivados del azufre. Por el contrario, en algunos hornos y en general en todo tipo de calderas las impurezas del combustible pueden ejercer efectos muy nocivos: alteraciones en la carga, corrosiones en los haces tubulares a elevada y baja temperatura, etc.

El contenido en asfaltenos de un fuel-oil no tiene importancia cuando el combustible se utiliza en hornos de gran radiación en los que la llama puede presentar notables longitudes que permitan la combustión completa de estos productos a su paso por la misma. Por el contrario, en hogares más fríos, y en general en cualquier tipo de calderas, un contenido elevado de asfaltenos implica mayor tendencia a la formación de humos, necesidad de trabajar con mayor exceso de aire y de multiplicar las limpiezas de hollines y, en definitiva, más contaminación y menor rendimiento. 


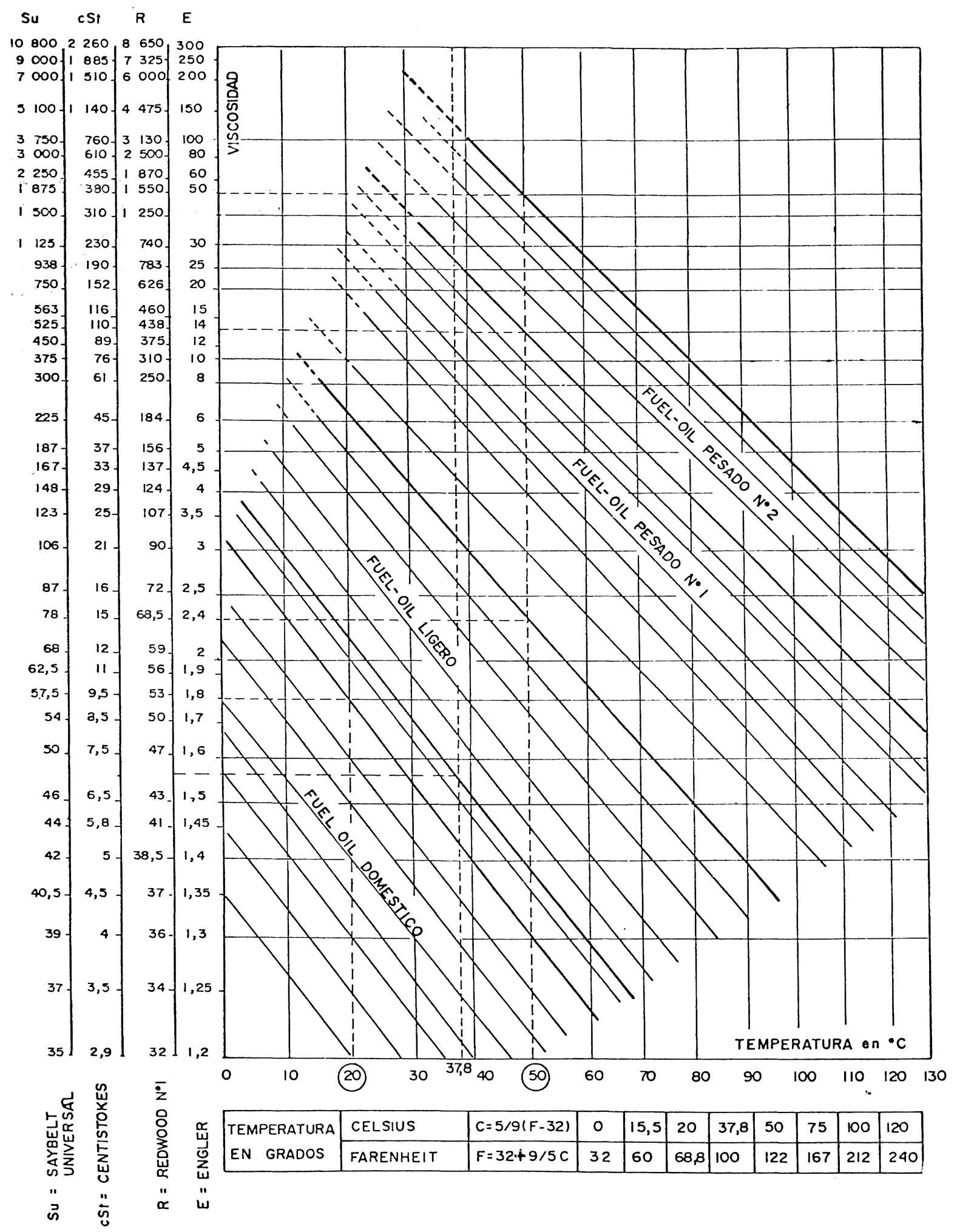

Fig. 5

Dentro de la terminología aceptada casi universalmente se vienen considerando como fuels pesados n." 2 aquellos de estos combustibles cuya viscosidad Engler a $50^{\circ}$ es superior a 14 (Fig. 5). Y es que a partir de estas viscosidades la pulverización y combustión 
del fuel va siendo progresivamente cada vez más difícil para los millares de equipos consumidores de mediana y pequeña potencia que trabajan en la industria y el sector doméstico. Asimismo, las fracciones de un fuel de viscosidad superior a la anteriormente citada, con independencia del origen del crudo y del proceso de refino, se van cargando progresivamente de impurezas y de derivados asfalténicos, lo que crea dificultades a un elevado número de utilizadores.

El aprovechamiento racional de los fuels residuales exige, pues, su fraccionamiento en las calidades cuyas especificaciones figuran en las tablas II, III y IV y en precios acordes con la calidad de las fracciones. La industria del cemento, con un consumo superior a $2,1 \times$ $\times 10^{6}$ t/año, así como otras actividades industriales capaces de utilizar fuels de inferior calidad, se deberían beneficiar por este hecho de precios más moderados de los que habrían de satisfacer los industriales que requieren combustibles de mejor calidad. Al mismo tiempo éstos últimos, aún abonando precios más elevados, disfrutarían la ventaja de disponer de un fuel más homogéneo, combustionable con rendimientos térmicos netamente superiores a los actuales y con una mayor vida de sus equipos de calor.

\section{RASGoS Fisico-quimicos DEL PROCESO DE FABRICACION DEL CEMENTO}

La moderna industria del cemento es inició a principios de siglo con la aparición del horno rotativo y del molino tubular. En los últimos años se han introducido notables progresos en la automatización de las instalaciones y ha venido creciendo continuamente tanto el tamaño como el número de los hornos rotativos que han, prácticamente, desplazado a los verticales.

Desde el punto de vista de la utilización de un combustible determinado para la producción de clínker y con independencia del procedimiento de fabricación adoptado, los fenómenos físicos y químicos que realmente nos interesa considerar son los incluidos en el recinto limitado por la línea de trazos de la figura 6 y que abarcan en esencia:

- el secado,

- la descarbonatación,

- la clinkerización,

- la agregación de aditivos previa a la molienda final del clínker,

ya que es en el área indicada donde se produce un íntimo contacto entre la carga y los gases de la combustión. Si incluimos los aditivos, es porque parte de los elementos contenidos en el combustible pasan al clínker y en mayor o menor medida podrían afectar el porcentaje de éstos útlimos.

Si despreciamos, por el momento, la contribución de elementos químicos que aparecen en reducidas proporciones en los crudos normales, tales como el magnesio, potasio, sodio y titanio - aunque como veremos más adelante su presencia en el crudo o en los cumbustibles tenga efectos notables sobre los procesos físicos y químicos que se desarrollan en el horno-, un balance típico de masas puede ser el representado en la figura 7.

En este balance ideal sólo se incluye el agua de constitución de las arcillas, pero no la humedad del crudo de alimentación que puede oscilar dentro de límites muy amplios en función del procedimiento de fabricación y de las características de las materias primas. 
Como valores orientativos del contenido en agua y para crudos típicos pueden tomarse los siguientes, referidos al momento de su entrada al secado:

- un $40 \%$ en el procedimiento por vía húmeda;

- un $20 \%$ en el procedimiento por vía semihúmeda;

- un $15 \%$ en el procedimiento por vía semiseca;

- un $8 \%$ en el procedimiento por vía seca.

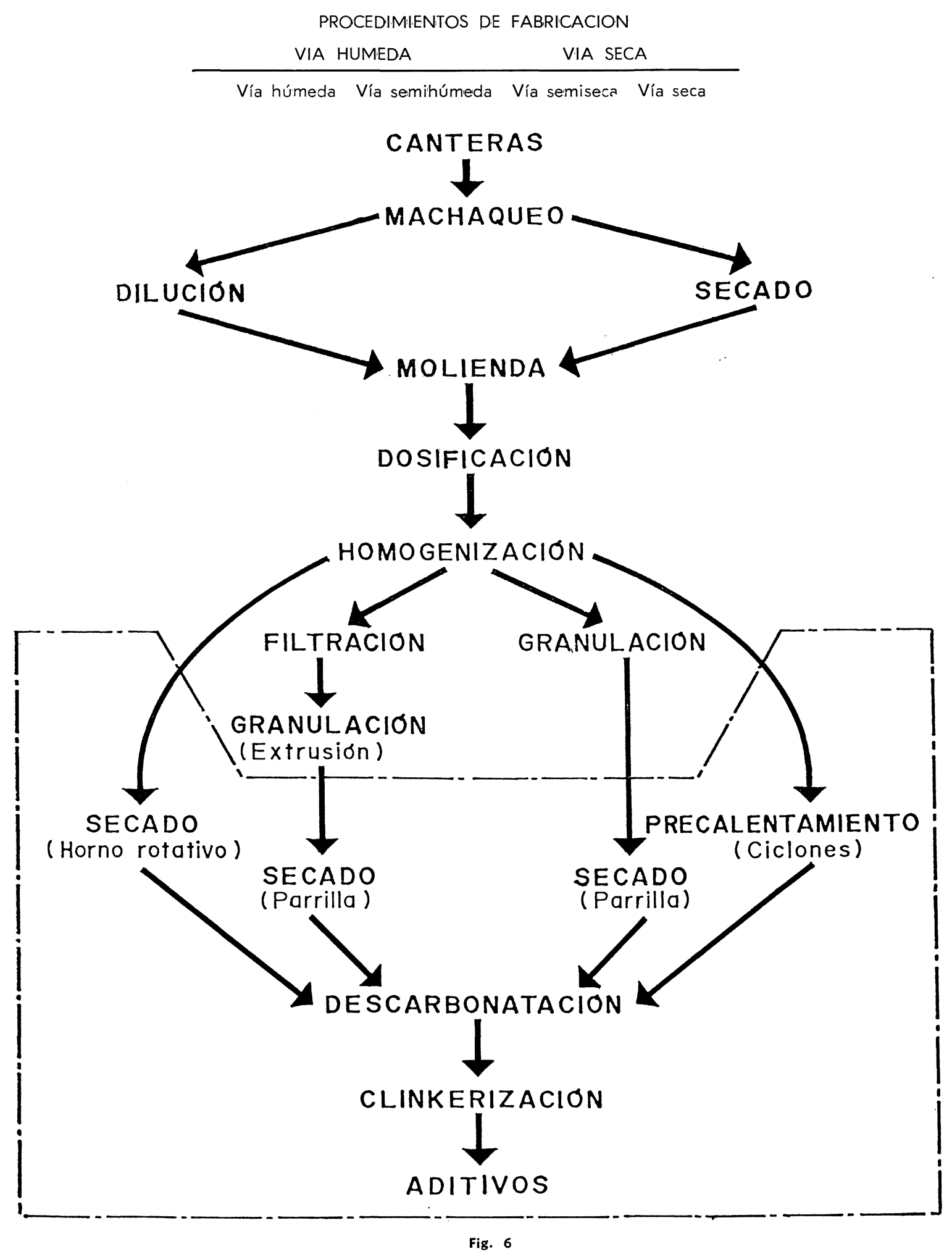


La fase gaseosa a través de las distintas secciones de un mismo horno o del horno y parrillas o ciclones de precalentamiento varía notablemente en composición química como consecuencia del desprendimiento, a diferentes temperaturas, del $\mathrm{CO}_{2}$ de la caliza, del agua de constitución de los materiales arcillosos y de la humedad del crudo. Asimismo resultan muy diferentes las composiciones de los gases según el procedimiento de fabricación y el tipo de combustible empleado. El contenido en vapor de agua aumenta naturalmente en los procesos por vía húmeda en relación con los de vía seca, pero lo hace asimismo en porcentajes apreciables al pasar de un carbón mineral a un fuel-oil residual o al gas natural como combustible.

REACTIVOS

\begin{tabular}{|c|}
\hline Compuestos \\
\hline 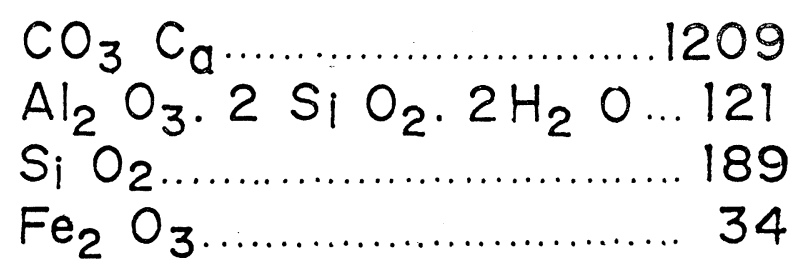 \\
\hline
\end{tabular}

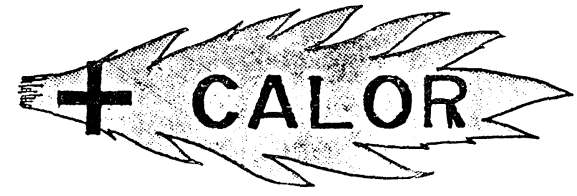

CLINKER

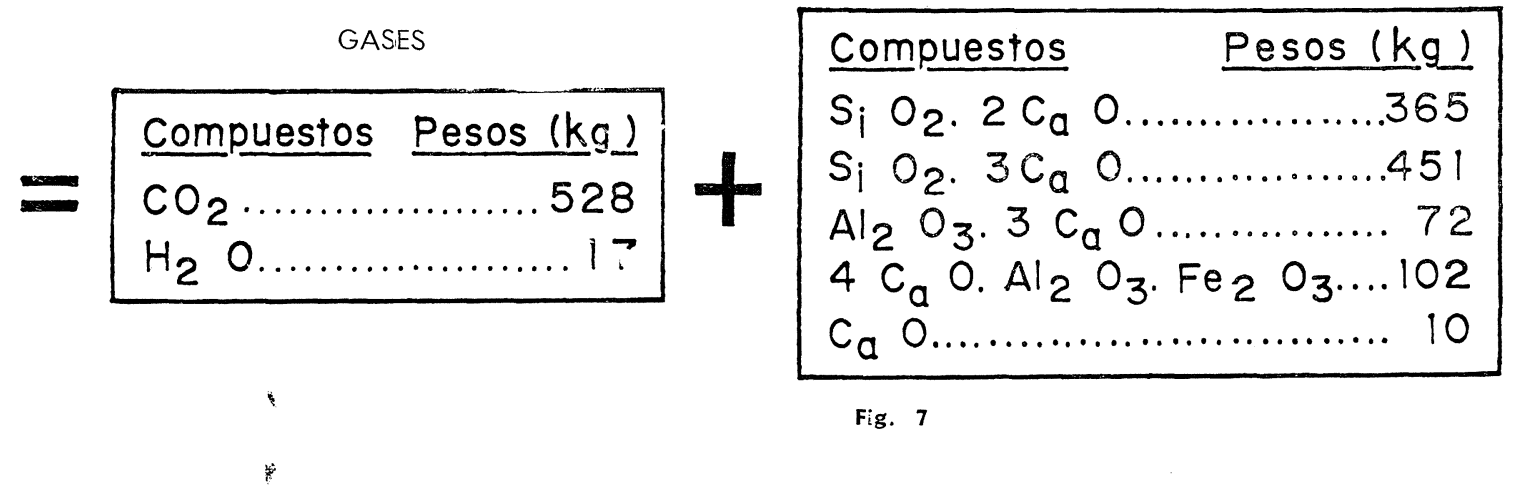

Los álcalis, aunque normalmente se encuentran en bajos porcentajes tanto en el crudo como en los combustibles, ejercen sus efectos físicos y químicos sobre el funcionamiento del horno. En los crudos pueden presentarse concentraciones de $\left(\mathrm{Na}_{2} \mathrm{O}+\mathrm{K}_{2} \mathrm{O}\right)$ superiores al $2 \%$. Las cenizas de algunos carbones minerales presentan asimismo concentraciones $\left(\mathrm{K}_{2} \mathrm{O}+\mathrm{Na}_{2} \mathrm{O}\right)$ de hasta más del $5 \%$. En los fuels derivados del petróleo, incluso en las fracciones residuales más viscosas comprendidas en un fuel pesado, la concentrạión en álcalis es como mucho de algunos centenares de ppm, por lo que su contribución puede considerarse despreciable en comparación con los álcalis aportados por la carga del horno.

El perfil de temperaturas a lo largo del eje de un horno rotativo es de difícil determinación. En la figura 8 se ilustra, según Weber, la variación de temperaturas de los gases y del material a lo largo de un proceso por vía seca con intercambiadores de precalentamiento de la harina. En el proceso de fabricación de clínker existen dos zonas principales:

- la de elevada temperatura, que comprende las zonas de sinterización y calcinación;

- la de baja temperatura, que abarca las zonas de prealentamiento y desecado del crudo. 
A partir de unos $650^{\circ} \mathrm{C}$ la carga del horno empieza a descarbonatarse. Desde esta temperatura hasta alcanzar aproximadamente $\operatorname{los} 1.500^{\circ} \mathrm{C}$ se van completando gradualmente los procesos de descarbonatación y clínkerización. En la figura 8 se señalan asimismo los contenidos en álcalis a lo largo del horno y, como se pone de manifiesto, éstos alcanzarán su máxima concentración a la entrada al horno rotativo.

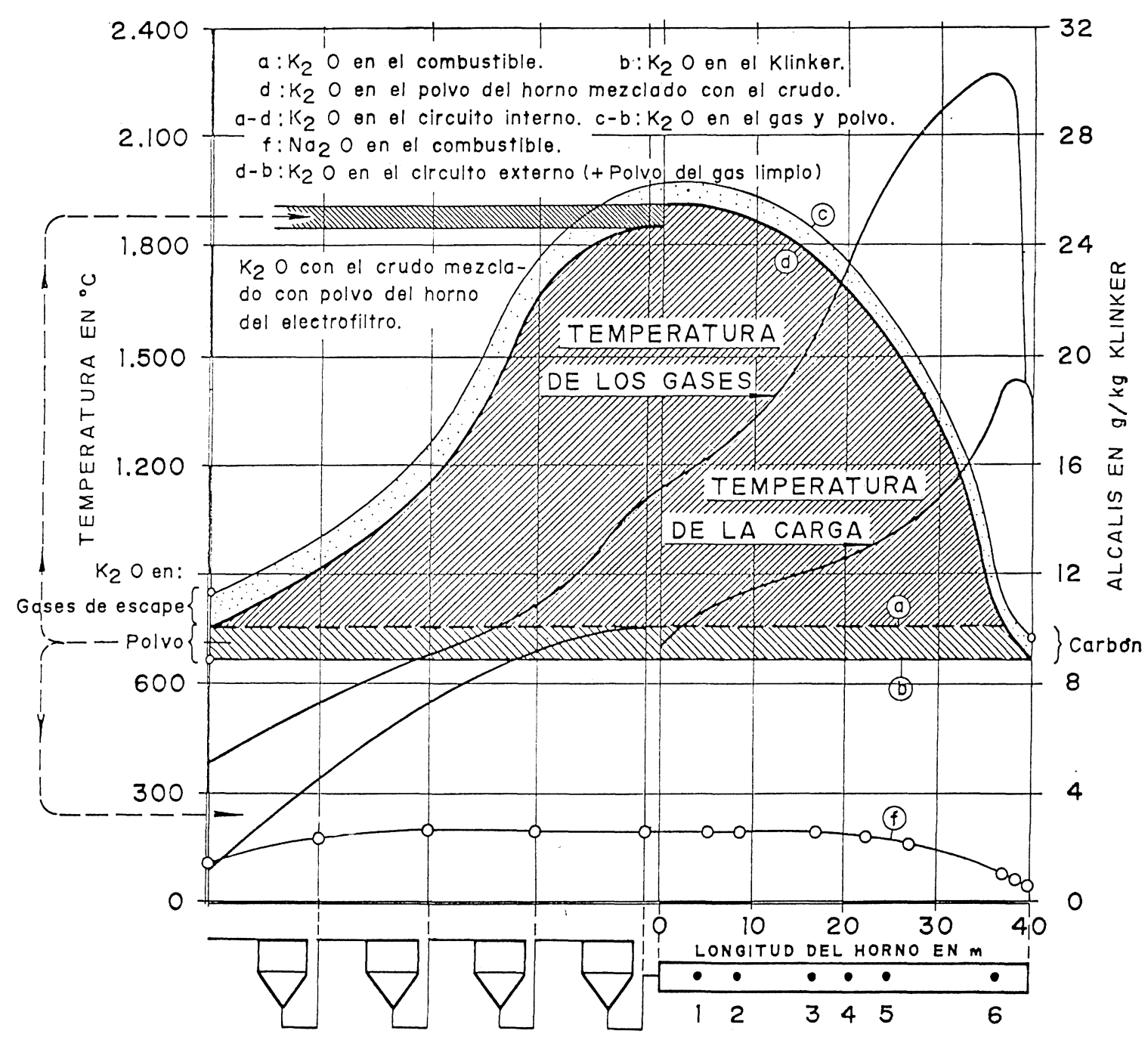

Fig. 8

El ciclo de los álcalis en las fábricas de cemento fue estudiado detenidamente por Weber (fig. 9) y otros autores. Los álcalis se evaporan parcialmente del crudo sometido a cocción, principalmente en la zona de sinterización, y se combinan con los aniones existentes en los gases formando sulfatos, carbonatos y cloruros. Salen del horno con los gases de escape y vuelven a condensarse a temperaturas inferiores a $900^{\circ} \mathrm{C}$, restituyéndose a la carga y desplazándose nuevamente a la zona de sinterización donde vuelven a evaporarse parcialmente. 
$\mathrm{El} \mathrm{SO}_{2}$ y el $\mathrm{SO}_{3}$, que pueden proceder tanto de la descomposición de los sulfatos presentes en el crudo como de la oxidación de los compuestos azufrados del combustible, forman sulfatos alcalinos de elevado punto de fusión $\left(1.075^{\circ} \mathrm{C}\right)$ y. difícilmente volatizables, por lo que en proporciones más o menos importantes pasan al clínker.

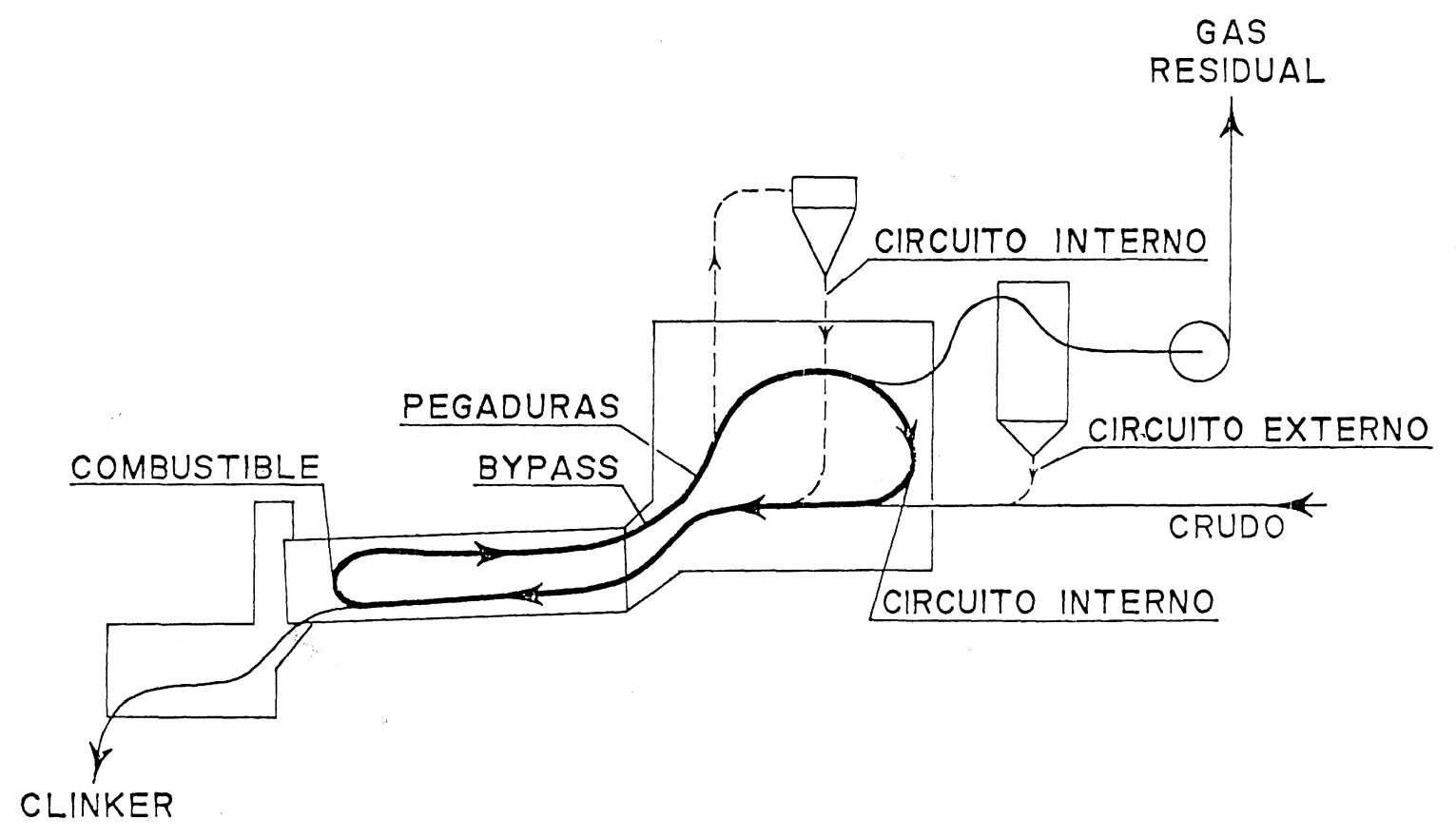

Fig. 9

El vapor de agua presente en los gases del horno y que procede tanto de la combustión del hidrógeno del combustible como de la evaporación del agua de constitución y de la humedad del crudo provoca la formación de hidróxidos alcalinos volátiles.

Los álcalis, en determinadas circunstancias de operación, forman pegaduras o costras que pueden perturbar las condiciones de tiro y dificultar la marcha del horno. Por otra parte, la evaporación de los álcalis se traduce siempre en el consumo de un calor de alta calidad en la zona de sinterización para ser devuelto en forma de energía de inferior calidad, en el momento de su condensación, lo que en cualquier caso implica un incremento en el consumo energético.

Los álcalis evacuados con el clínker pueden afectar al fraguado del cemento si se convierten en carbonatos alcalinos por ácción del $\mathrm{CO}_{2}$ del aire. Sin embargo, la experiencia demuestra que los álcalis en forma de sulfatos no ejercen influencia alguna sobre el fraguado.

Si los álcalis pasan al clínker en forma de silicatos o aluminatos alcalinos $\left(\mathrm{S}_{12} \mathrm{C}_{23} \mathrm{~K}, \mathrm{~A}_{3} \mathrm{C}_{8} \mathrm{~K}\right.$ y $\mathrm{A}_{3} \mathrm{C}_{8} \mathrm{Na}$ ), tendencia que se ve favorecida por el incremento en el vapor de agua en los gases del horno, se combina menos $\mathrm{CaO}$, por lo que no podrá trabajarse con un grado de saturación en cal demasiado elevado porque resultaría una cantidad excesiva de cal libre. 
Tanto en el horno rotativo como en los sistemas de recuperación de calor -parrillas y ciclones-, se producen interaciones químicas entre los gases y la carga del horno. Estas interacciones se ven favorecidas por tres factores:

- las elevadas temperaturas de la carga y de los gases;

- el ambiente pulverulento que reina en el interior del horno favorece un íntimo contacto entre los gases y la fase sỏlida en suspensión que presenta una gran superficie específica;

- el tiempo de contacto entre los gases y la carga es muy elevado a lo que contribuye la gran longitud del horno y el intenso reciclado de la fase sólida en suspensión.

Como consecuencia de lo dicho anteriormente puede afirmarse que los efectos químicos de utilizar un fuel pesado n. $^{\circ} 2$, en lugar del actual fuel, sobre la marcha del horno serían los siguientes:

- se produciría un mayor volumen de $\mathrm{SO}_{2}$ y de $\mathrm{SO}_{3}$ en la combustión;

- este mayor porcentaje de $\mathrm{SO}_{2}$ y $\mathrm{SO}_{3}$, permaneciendo iguales las demás condiciones, disminuirá la volatilidad de los álcalis e incrementará la proporción de los mismos que pasarán al clínker como sulfatos;

- la cantidad de álcalis existentes en el crudo y el $\mathrm{CaO}$ procedente de la descarbonatación de la cal, así como las temperaturas y el tiempo de contacto de estas sustancias con los óxidos de azufre, garantizan su combinación completa con los óxidos alcalinos y su paso a sulfatos de manera que, en régimen estacionario, no podrá detectarse la existencia de $\mathrm{SO}_{2}$ ni de $\mathrm{SO}_{3}$ en los gases de chimenea.

El horno de cemento constituye, pues, una de las instalaciones desulfuradoras de gases más eficientes, y por eso está llamado a utilizar fuels con porcentajes de impurezas que los hacen inaceptables o poco idóneos en otras aplicaciones.

Los problemas físicos que se derivan de la sustitución del combustible se deben a la mayor viscosidad del fuel pesado $\mathrm{n}^{\circ} 2 \mathrm{y}$ a su más elevado contenido en asfaltenos.

Los efectos de la mayor viscosidad, que se traducirían en una pulverización más grosera del combustible, pueden normalmente contrarrestarse en los sistemas de preparación del fuel como veremos más adelante.

Pero, incluso manteniendo la calidad de la pulverización del incremento en el contenido de asfaltenos, tenderá a dar llamas más radiantes y de longitud superior. Los efectos negativos que pudieran achacarse a estos fenómenos pueden corregirse normalmente en el quemador como veremos después.

\section{TRANSPORTE, ALMACENAMIENTO Y COMBUSTION DEL FUEL PESAdo $\mathbf{N} .^{\circ} 2$}

Para finalizar quisiera hacer una breve referencia de los problemas que podría plantear, en principio, el transporte, almacenamiento y combustión del fuel pesado n. ${ }^{\circ} 2$.

\subsection{Transporte}

En este aspecto cabe considerar diversas circunstancias en función de los medios normales de suministro, volúmenes de consumo y situación geográfica de las fábricas de cemento. 
Las fábricas conectadas directamente por tubería, aislada y calorifugada, a las Factorías de CAMPSA no tendrían, en principio, dificultad para recibir este fuel. Posiblemente, por cuestión de economía en la energía de bombeo o de resistencia mecánica de las tuberías, se haría conveniente o necesario en algún caso elevar la temperatura del fluido a transportar; esto podría representar algún pequeño gasto complementario de energía para la fábrica, siempre que ésta tenga a su cargo el calorifugado de la línea. En el mismo caso se encuentran las fábricas que disponen de terminales marítimas con tuberías aisladas y calorifugadas.

El medio de suministro más generalizado es por vagones cisternas constituyendo trenes puros. Posiblemente buena parte del parque de vagones no cuentan con serpentines de calefacción de suficiente superficie. Este es fundamentalmente un problema de CAMPSA, aunque podría afectar a la fábrica si no cuenta con la suficiente capacidad de fluido de caldeo. Por término medio el paso del fuel actual al pesado requiere un calentamiento complementario del combustible de unos $15^{\circ} \mathrm{C}$ para su descarga de los trenes a los tanques de almacenamiento, su trasiego de éstos últimos a los nodriza y su precalentamiento en el sistema de preparación del combustible para lograr las mismas condiciones hidráulicas y de combustión. El importe total de este gasto de energía complementario, para un almacenamiento de $10.000 \mathrm{~m}^{3}$ y un consumo mensual de $7.000 \mathrm{t}$, puede fijarse entre diez y quince pesetas por tonelada de fuel consumido, según el clima de la localidad.

En cuanto al transporte por camiones cisternas, medio de suministro también utilizado con frecuencia, puede estimarse que la flota actual, sin aislamiento ni calorifugado no es idónea para el transporte de fuel pesado.

\subsection{Almacenamiento}

Los actuales tanques de fuel-oil cuyos serpentines de fondo y calentadores de succión se hayan diseñado con cierta holgura se podrán utilizar normalmente para almacenar el fuel pesado $n .^{\circ} 2$.

No obstante, ante la posibilidad de que se originen dificultades por el incremento en la viscosidad del combustible, conviene asegurarse previamente de la capacidad de los elementos de calefacción del tanque, recurriendo, en caso de duda, al asesoramiento de técnicos especializados en estas cuestiones.

\subsection{La combustión del fuel pesado $n .^{\circ} 2$}

Actualmente se encuentran en el mercado quemadores de llama regulable en marcha y de potencia variable que pueden alimentarse con fuels residuales de cualquier característica y que permiten disminuir el aire primario y frío, necesario para la pulverización y la refrigeración de la caña, a valores muy reducidos - hasta el $5 \%$ del estequiométrico-, con lo que se incrementan notablemente las posibilidades de aprovechamiento del calor sensible al clínker calentando el aire secundario de combustión. Asimismo, por la notable longitud alcanzada por los cañones de los quemadores, los hornos pueden acomodarse a diversos regímenes de funcionamiento y la zona de sinterización puede desplazarse o modificarse.

El cambio de un fuel híbrido, aunque de mediana viscosidad, como el actual, por el de tipo pesado n. ${ }^{\circ} 2$ se traduciría, si no se realizan las oportunas regulaciones, en una pulverización más grosera que tendería a incrementar las dimensiones de la llama. En este 
mismo sentido actuaría la diferencia en la composición química del combustible, ya que las gotas con gran contenido en asfaltenos requieren mayor tiempo para su combustión completa que las constituidas por hidrocarburos de menor peso molecular.

No obstante, la dificultad apuntada se puede contrarrestar satisfactoriamente si se dispone de un conjunto quemador/sistema de preparación del combustible diseñado con cierta holgura. La combustión completa y adecuada de un fuel pesado n. ${ }^{\circ} 2$ podrá conseguirse con todas o algunas de las siguientes medidas:

- incrementando moderadamente (hasta unos $15^{\circ} \mathrm{C}$ como máximo) la temperatura de precalentamiento del fuel;

— incrementando la presión de inyección del fuel asimismo de manera moderada;

— incrementando ligeramente la presión y el caudal del aire primario.

Puede decirse, como resumen, que el cambio de combustible lleva aparejados unos efectos sobre la combustión que pueden ser contrarrestados por los modernos equipos que cuentan con suficiente flexibilidad. En cualquier caso y previamente al cambio conviene consultar con especialistas en esta materia.

T A B L A II

Especificaciones del fuel-oil pesado número $1\left({ }^{*}\right)$

\begin{tabular}{|c|c|c|c|c|c|}
\hline \multirow{2}{*}{ Características } & \multirow{2}{*}{$\begin{array}{l}\text { Unidades } \\
\text { de medida }\end{array}$} & \multicolumn{2}{|c|}{ Límites de cspecificación } & \multirow{2}{*}{$\begin{array}{l}\text { Normas } \\
\text { INTA }\end{array}$} & \multirow{2}{*}{$\begin{array}{l}\text { Normas } \\
\text { ASTM }\end{array}$} \\
\hline & & Mínimo & Máximo & & \\
\hline a. Color negro & - & - & - & - & - \\
\hline b. Viscosidad $50^{\circ} \mathrm{C}$ (1) & ${ }^{\circ} \mathrm{E}$ & 7 & 14 & 150218 & - \\
\hline c. Azufre & $\%$ peso & - & 2,5 & 150441 & $D-1551$ \\
\hline d. Punto de inflamación & ${ }^{\circ} \mathrm{C}$ & 70 & - & $150234 \mathrm{~B}$ & $\begin{array}{l}D-1552 \\
D-93\end{array}$ \\
\hline e. Agua y sedimento & $\%$ vol. & - & 1,0 & $150462 C$ & D-1796 \\
\hline f. Agua & $\%$ vol. & - & 0,75 & $150456 \mathrm{~A}$ & D-95 \\
\hline & & & & $150439 B$ & $D-129$ \\
\hline g. Potencia calorífica & & & & & \\
\hline - Superior & $\mathrm{Kcal} / \mathrm{kg}$ & 10.200 & - & & $D-210$ \\
\hline - Inferior & $\mathrm{Kcal} / \mathrm{kg}$ & 9.700 & - & & $D-240$ \\
\hline
\end{tabular}

(*) Cuando el contenido en azufre de esta calidad no supere el 1 por 100 en peso, se denominará fuel-oil número 1 BIA, y cuando no supere el 0,6 por 100, se denominará fuel-oil número 1 BIA especial.

Los suministros de estos tipos de combustibles con bajo contenido de azufre serán regulados por el Ministerio de Industria y la forma de distribución será fijada por la Delegación del Cobierno en CAMPSA.

(1) Para esta determinación se pueden utilizar también los métodos INTA 150216A y 150217A, que corresponden a los ASTM D-445 y D-280 respectivamente, calculando mediante tablas los ${ }^{\circ} \mathrm{E}$ correspondientes. 
T A B L A III

Especificaciones del fuel-oil pesado número 2

\begin{tabular}{|c|c|c|c|c|c|}
\hline \multirow{2}{*}{ Características } & \multirow{2}{*}{$\begin{array}{l}\text { Unidades } \\
\text { de medida }\end{array}$} & \multicolumn{2}{|c|}{ Límites de especificación } & \multirow{2}{*}{$\begin{array}{l}\text { Normas } \\
\text { INTA }\end{array}$} & \multirow{2}{*}{$\begin{array}{l}\text { Normas } \\
\text { ASTM }\end{array}$} \\
\hline & & Mínimo & Máximo & & \\
\hline a. Color negro & - & - & - & - & - \\
\hline b. Viscosidad $50^{\circ} \mathrm{C}$ (1) & ${ }^{\circ} \mathrm{E}$ & - & 50 & 150218 & - \\
\hline c. Azufre & $\%$ peso & - & 3,6 & 150439B & $D-1<9$ \\
\hline d. Punto de inflamación & ${ }^{\circ} \mathrm{C}$ & 70 & & & 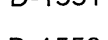 \\
\hline e. Aqua y sedimento & $\%$, & $\ldots$ & 175 & $150231 \mathrm{R}$ & 500 \\
\hline c & & & & 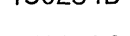 & \\
\hline f. Agua & $\%$ vol. & - & 1,5 & $150412 C$ & D-1796 \\
\hline g. Potencia calorífica & & & & $150456 \mathrm{~A}$ & D-95 \\
\hline - Superior & $\mathrm{Kcal} / \mathrm{kg}$ & 10.000 & - & & $D-240$ \\
\hline - Inferior & $\mathrm{Kcal} / \mathrm{kg}$ & 9.500 & - & & D-240 \\
\hline
\end{tabular}

(1) Para esta determinación se pueden utilizar también los métodos INTA 150216A y 150217A, que corresponden a los ASTM D-445 y D-294 respectivamente, calculando mediante tablas los oE correspondientes.

T A в L A IV

Especificaciones de carbones para calefacciones y para usos industriales

(Porcentaje en peso)

CALEFACCIONES Y USOS DOMESTICOS (*)

CALIDAD NUMERO 1 , UTILIZABLE EN ZONAS

DE ATMOSFERA CONTAMINADA

\begin{tabular}{l|l|l}
\hline & Antracita & $\begin{array}{c}\text { Aglomerados } \\
\text { sin humos }\end{array}$ \\
\cline { 2 - 3 } & $\leqslant 1$ & $\leqslant 1$ \\
$\begin{array}{l}\text { Azufre } \\
\text { Celátiles }\end{array}$ & $\sum 10$ & $\sum 10$ \\
$\begin{array}{l}\text { Humizas } \\
\text { Granulad } \\
\text { tría }\end{array}$ & $<12$ & $<12$ \\
& $<15 \mathrm{~mm}$ & $<1,5$
\end{tabular}

CALIDAD NUMERO 2, UTILIZABLE EN ZONAS DE ATMOSFERA EN SITUACION ADMISIBLE

\begin{tabular}{|c|c|c|c|}
\hline & Antracita & $\begin{array}{l}\text { Hulla baja en } \\
\text { volátiles }\end{array}$ & Aglomerados \\
\hline $\begin{array}{l}\text { Azufre } \\
\text { Volátiles } \\
\text { Cenizass } \\
\text { Humedad } \\
\text { Granulome- } \\
\text { tría }\end{array}$ & $\begin{array}{l}\leqslant 1,2\left({ }^{* *}\right) \\
<10^{\circ} \\
<15 \\
8 \\
>10 \mathrm{~mm}\end{array}$ & $\begin{array}{l}\leqslant 1,2\left({ }^{* *}\right) \\
<15 \\
\sum 15 \\
<8 \\
>10 \mathrm{~mm}\end{array}$ & $\begin{array}{l}\leqslant 1,2\left({ }^{* *}\right) \\
<15 \\
<15 \\
8 \\
-\end{array}$ \\
\hline
\end{tabular}

(*) En determinadas zonas y en circunstancias especiales, el Ministerio de Industria podrá utilizar para estos usos el consumo de carbones no comprendidos en la anterior clasificación.

(**) Azufre pirítico. 


\section{CONCLUSIONES}

Podemos resumir lo expuesto en esta comunicación en los siguientes puntos:

- La política de energía excesivamente barata ocasionó perjuicios a la nación porque alentó el despilfarro energético y desaceleró el desarrollo de los recursos energéticos propios; esta política no puede ser tolerada por más tiempo por nuestra economía.

- Es contraproducente seguir manteniendo en el mercado nacional un solo tipo de fueloil indiferenciado en calidad y precio, porque las posibilidades y necesidades de los distintos sectores consumidores están muy lejos de ser similares.

- Las industrias que como las del cemento consumen grandes cantidades de combustible, que podrían ser de calidad sensiblemente inferior a la del actual, habrían de beneficiarse de esta circunstancia disfrutando de precios inferiores a los de otras actividades industriales que necesitan combustibles de mejor calidad y cuya incidencia en el coste del producto acabado es en ocasiones muy reducida.

- Parecería aconsejable que los recursos que viene destinando la Administración para bonificar el precio de algunas energías se dirigieran a ayudar a aquellas industrias que estuviesen formalmente comprometidas a mejorar sus procesos e instalaciones para un mejor aprovechamiento de la energía; tal medida redundaría además en la reactivación de sectores industriales muy precisados de ella. 